\title{
Effects of intubation with a double-lumen endotracheal tube on intraocular pressure during rapid sequence induction using succinylcholine chloride in patients with or without underlying systemic hypertension
}

Received May 2, 2019

Revised 1st, July 22, 2019

2nd, July 29, 2019

3rd, July 31, 2019

Accepted August 1, 2019

\section{Corresponding author}

Jaemin Lee, M.D., Ph.D.

Department of Anesthesiology and

Pain Medicine, Uijeongbu St. Mary's

Hospital, The Catholic University

of Korea, College of Medicine, 271

Cheonbo-ro, Uijeongbu 11765,

Korea

Tel: 82-31-820-3256

Fax: 82-31-847-3449

E-mail: jmlee@catholic.ac.kr

ORCID

https://orcid.org/0000-0001-8281-5622

\section{Chan-oh Park', Hojun Ro ${ }^{2}$, and Jaemin Lee ${ }^{2}$}

Department of Anesthesiology and Pain Medicine, 'Seoul St. Mary's Hospital, College of Medicine, The Catholic University of Korea, Seoul, ${ }^{2}$ Uijeongbu St. Mary's Hospital, The

Catholic University of Korea, College of Medicine, Uijeongbu, Korea

Background: Tracheal intubation is closely associated with increases in intraocular pressure (IOP); however, the effects of double-lumen tube (DLT) intubation on IOP have not been validated. Systemic hypertension (HTN) is another factor that may increase IOP. In this study, we observed differences in IOP increases between DLT and singlelumen tube (SLT) intubation, and evaluated the influence of underlying HTN during rapid sequence induction.

Methods: Sixty-eight patients were allocated into one of the following group: SLT/without HTN ( $n=17)$, SLT/HTN ( $n=17)$, DLT/without HTN $(n=17)$, and DLT/HTN ( $n=17)$. An SLT was inserted for orthopedic or gynecological surgery, and a DLT was inserted for lung surgery after rapid sequence induction using succinylcholine. IOP was measured before anesthetic induction and until 10 min after intubation using a handheld tonometer (Tono-Pen AVIA ${ }^{\circledR}$ ).

Results: In the DLT/without HTN and DLT/HTN groups, the maximum increases in IOPs after tracheal intubation were 7.9 and $12.2 \mathrm{mmHg}$, respectively, compared to baseline. In the SLT/without HTN and SLT/HTN groups, the maximum increases were 5.0 and 4.9 $\mathrm{mmHg}$, respectively, compared to baseline. In comparisons between patients with and without underlying HTN, the values of IOPs were comparable.

Conclusions: Tracheal intubation with a DLT is associated with more increases in IOPS than with an SLT in rapid sequence induction. Well-controlled underlying hypertension did not increase IOP during tracheal intubation.

Keywords: Double-lumen tracheal tube; Hypertension; Intraocular pressure; Intubation, intratracheal.

\section{INTRODUCTION}

Tracheal intubation is closely associated with increased intraocular pressure (IOP) [1]. For lung surgeries, such as lobectomy or wedge resection, it is imperative to place a double-lumen tube (DLT) to perform one-lung ventilation. The external diameter of the DLT is larger than that of a single-lumen tube (SLT), therefore, intubation with a DLT is considered more difficult, more time consuming, and more stimulating to the trachea, which may lead to higher sympa-

This is an Open Access article distributed under the terms of the Creative Commons Attribution Non-Commercial License (http://creativecommons.org/licenses/by-nc/4.0) which permits unrestricted non-commercial use, distribution, and reproduction in any medium, provided the original work is properly cited. 
thetic responses than intubation with an SLT $[2,3]$. Considering the magnitude of the stimuli, tracheal intubation using a DLT may cause a greater increase in IOP than that using SLT. However, the relationship between DLT placement and changes in IOP has not been elucidated.

An additional factor that may influence IOP is systemic hypertension (HTN). In animal studies, the relationship between HTN and IOP has been established as HTN being one of the major causes of glaucoma [4]. However, it has not yet been validated in humans undergoing tracheal intubation. Because of an increase in the prevalence of HTN and a subsequent growing trend in the frequency of surgery in hypertensive patients, it is important to compare the changes in IOP between normotensive patients and hypertensive patients during tracheal intubation.

In the present study, we compared increases in IOPs during tracheal intubation using a DLT or SLT in patients with or without systemic HTN. We hypothesized that tracheal intubation with a DLT and patients with HTN would be associated with a greater increase in IOP than tracheal intubation with an SLT and patients without HTN. We performed a rapid sequence induction using succinylcholine simulating emergency intubation where the DLT and the underlying HTN could show a significant influence on IOP.

\section{MATERIALS AND METHODS}

The study was approved by the Institutional Review Board of the Seoul St. Mary's Hospital (no. KC16OISI0689). Each patient gave verbal and written informed consent in advance. Sixty-eight adult patients of the American Society of Anesthesiologist physical status class I or II, who were scheduled for general anesthesia with tracheal intubation between September 2016 and August 2017, were included in this controlled, prospective study. Patients were excluded if they had preexisting eye disease including glaucoma, previous eye surgery, preoperative unstable hemodynamics, or anticipated difficult tracheal intubation.

Patients were allocated into one of the following four groups: patients without HTN who were intubated using an SLT (SLT/without HTN group, $\mathrm{n}=17$ ), patients with HTN who were intubated using an SLT (SLT/HTN group, $n=17$ ), patients without HTN who were intubated using a DLT (DLT/ without HTN group, $n=17$ ), and patients with HTN who were intubated using a DLT (DLT/HTN group, $n=17$ ). Every patient with HTN was diagnosed by the medical staff and had been treated with antihypertensive agents before surgery. Patients with blood pressure $<140 / 90 \mathrm{mmHg}$ measured in the operating room were included. This blood pressure standard was equally applied to patients without a history of hypertension. The primary outcome was IOP after tracheal intubation using either an SLT or DLT, and secondary outcomes were IOP at other time points and ocular perfusion pressure (OPP), which was calculated as the mean arterial pressure (MAP) minus IOP, between patients with or without HTN.

Intraoperative monitoring included an electrocardiogram, noninvasive blood pressure, pulse oximetry, capnometry, bispectral index (BIS), and IOP. For anesthetic induction, 5 $\mathrm{mg} / \mathrm{kg}$ pentothal sodium, $1 \mathrm{mg} / \mathrm{kg}$ succinylcholine, and $1 \mu \mathrm{g} /$ $\mathrm{kg}$ remifentanil were administered as a formula for rapidsequence induction. The trachea was intubated after $60 \mathrm{~s}$ of manual ventilation with $100 \%$ oxygen. Intubation of the trachea was attempted with either a cuffed SLT with an internal diameter of $8 \mathrm{~mm}$ for males and $7.5 \mathrm{~mm}$ for females, or a leftbronchial DLT at a size of $37 \mathrm{Fr}$. for males and $35 \mathrm{Fr}$. for females. A stylet was used for all patients. The SLT was used for orthopedic or gynecological surgery, and the DLT was used for lung surgery. Anesthesia was maintained with end-tidal $1.5-2.0$ volume\% of sevoflurane and $60-300 \mu \mathrm{g} / \mathrm{h}$ of continuous infusion of remifentanil, maintaining the BIS at approximately 50 and the MAP between $\pm 20 \%$ of the baseline value. Controlled ventilation was maintained with air and oxygen $\left(\mathrm{FiO}_{2}: 0.5\right)$ to maintain the end-tidal carbon dioxide $\left(\mathrm{EtCO}_{2}\right)$ between 30-35 mmHg. Normosol-R was used for intraoperative fluid therapy.

IOP was measured using a handheld tonometer, Tono-Pen AVIA $^{\circledast}$ (Reichert Technologies, USA) [5]. After applying two drops of $0.5 \%$ proparacaine $\mathrm{HCl}$ (S.A. Alcon-Couvreur N.V.) for topical anesthesia, IOP was measured seven times: before anesthetic induction (baseline value, T1), after administration of anesthetic agents and before tracheal intubation (T2), right after tracheal intubation (T3), and 1, 3, 5, and $10 \mathrm{~min}$ after tracheal intubation (T4-T7). IOP measurements using portable tonometry required skilled techniques. All IOP measurements were made by one researcher who was not involved in the study and was experienced in using portable tonometry. At the time of each tonometer reading, the following data were collected: MAP, heart rate, and $\mathrm{EtCO}_{2}$. In the 
recovery room, patients were asked about any vision changes or eye discomfort.

\section{Statistics}

For sample size calculations, an independent $t$-test was performed to evaluate differences in IOP after tracheal intubation between the SLT/without HTN and DLT/without HTN groups. In a pilot study, the values of IOP showed a normal distribution as a continuous variable, and the difference in mean values between groups was $4 \mathrm{mmHg}$ with a standard deviation of 4.0. The sample size required at a level of significance of $5 \%$ (two-sided alpha $=0.05$ ) and a power of $80 \%$ ( 1 - beta $=0.8)$ was 17 patients per group. To compare demographic data, a chi-square test and independent $t$-test were used. IOP and OPP between groups were subjected to repeated-measures analysis of variance with "group" and "time point" as independent variables, after confirming a normal distribution of the data using the Shapiro-Wilk test. The interaction term was calculated using a Bonferroni correction for repeated measures. A P value $<0.05$ was considered to indicate statistical significance. All data were analyzed using PASW statistical software for Windows, version 18.0 (IBM Co., USA).

Table 1. Demographic Data

\begin{tabular}{lcccc}
\hline Variable & $\begin{array}{c}\text { Group without HTN/SLT } \\
(\mathrm{n}=17)\end{array}$ & $\begin{array}{c}\text { Group without HTN/DLT } \\
(\mathrm{n}=17)\end{array}$ & $\begin{array}{c}\text { Group HTN/SLT } \\
(\mathrm{n}=17)\end{array}$ & $\begin{array}{c}\text { Group HTN/DLT } \\
(\mathrm{n}=17)\end{array}$ \\
\hline Age $(\mathrm{yr})$ & $51.2 \pm 11.5$ & $53.4 \pm 8.6$ & $58.6 \pm 13.1$ & $57.9 \pm 12.9$ \\
Sex (M/F) & $8 / 9$ & $9 / 8$ & $7 / 10$ & $9 / 8$ \\
Weight $(\mathrm{kg})$ & $68.7 \pm 13.3$ & $66.4 \pm 12.5$ & $69.5 \pm 14.8$ & $63.8 \pm 15.5$ \\
Height $(\mathrm{cm})$ & $165.9 \pm 9$ & $169.4 \pm 10.2$ & $164.7 \pm 9.2$ & $169.1 \pm 10.5$ \\
MAP (mmHg) & $93.6 \pm 9.1$ & $95.1 \pm 8.6$ & $94.9 \pm 9.2$ & $99.6 \pm 10.9$ \\
HR (rate/min) & $83.2 \pm 8.6$ & $81.3 \pm 7.5$ & $79.2 \pm 9.2$ & $81.5 \pm 5.7$ \\
ASA PS (I/I) & $13 / 4$ & $12 / 5$ & $0 / 17$ & $0 / 17$ \\
\hline
\end{tabular}

Values are presented as mean \pm SD or number only. No significant difference was found between groups. HTN: high blood pressure, SLT: single lumen tube, DLT: double lumen tube, M: male, F: female, MAP: mean arterial pressure at preinduction, HR: heart rate at preinduction, ASA PS: American Society of Anesthesiologist physical status.

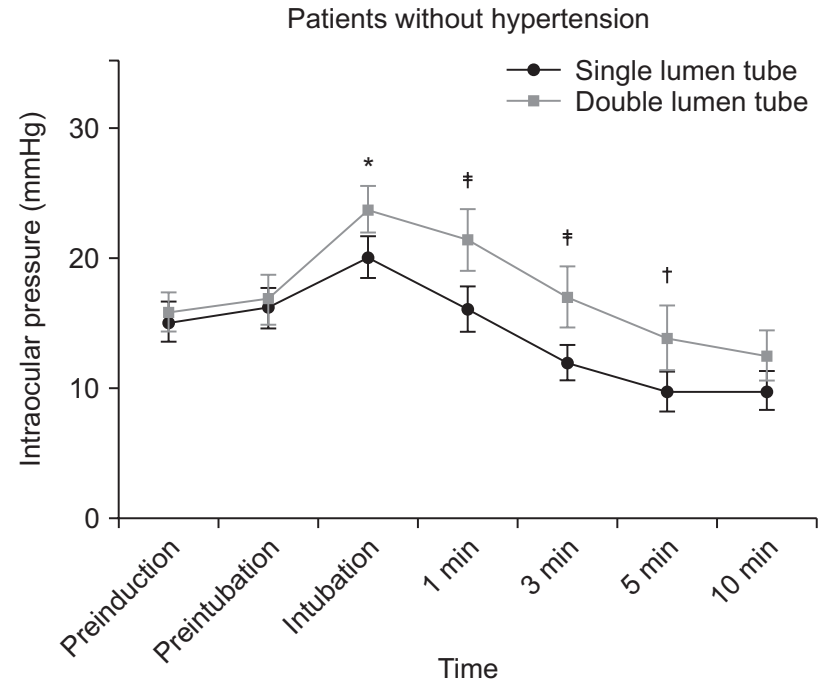

Fig. 1. Time course of intraocular pressure (IOP) for patients without hypertension. Patients who were intubated with double lumen tube showed higher IOPs immediately, 1, 3, and 5 min after tracheal intubation than those who were intubated with single lumen tube. Symbols and error bars indicate means and SD, respectively. ${ }^{*} \mathrm{P}<0.05,{ }^{\dagger} \mathrm{P}<$ $0.01,{ }^{\ddagger} \mathrm{P}<0.001$ compared to single lumen tube.

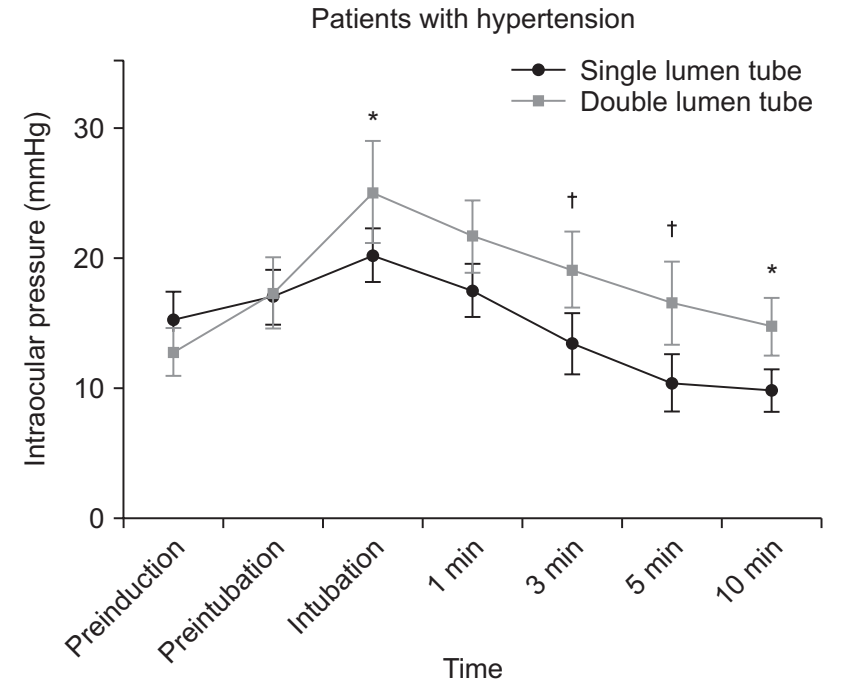

Fig. 2. Time course of intraocular pressure (IOP) for patients with hypertension. Patients who were intubated with double lumen tube showed higher IOPs immediately, 3, 5, and 10 min after tracheal intubation than those who were intubated with single lumen tube. Symbols and error bars indicate means and SD, respectively. ${ }^{*} P<0.05,{ }^{\dagger} P<0.01$ compared to single lumen tube. 


\section{RESULTS}

In total, 74 patients were recruited for the study. Five patients were excluded from data analyses due to violation of the study protocol by the anesthesiologist, and one patient was excluded due to an unexpected difficult intubation. Demographic data are shown in Table 1. No patients complained of any vision changes or eye discomfort in the recov-

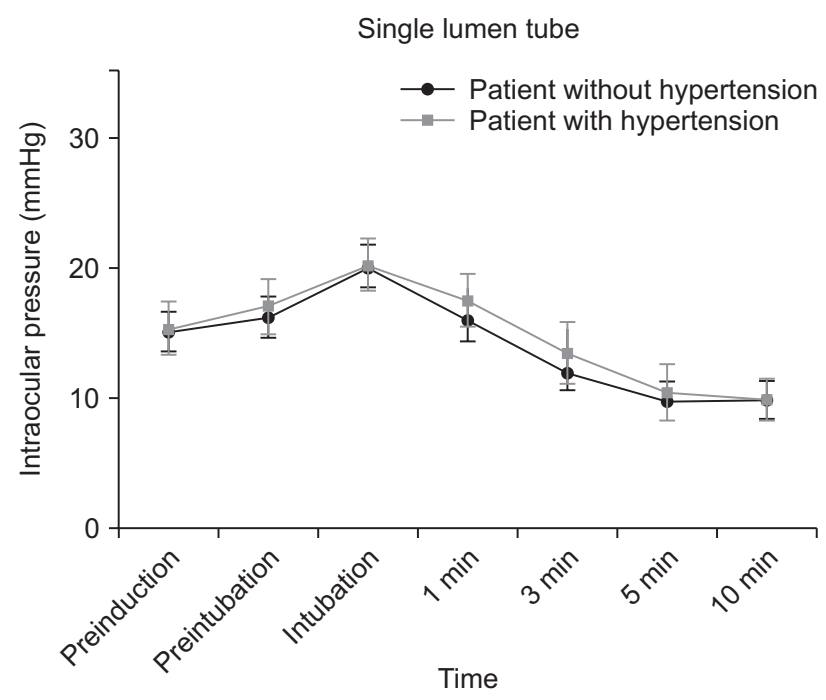

Fig. 3. Time course of intraocular pressure (IOP) for patients intubated with single lumen tube. There were no differences in IOPs between patients with and without hypertension. Symbols and error bars indicate means and SD, respectively.

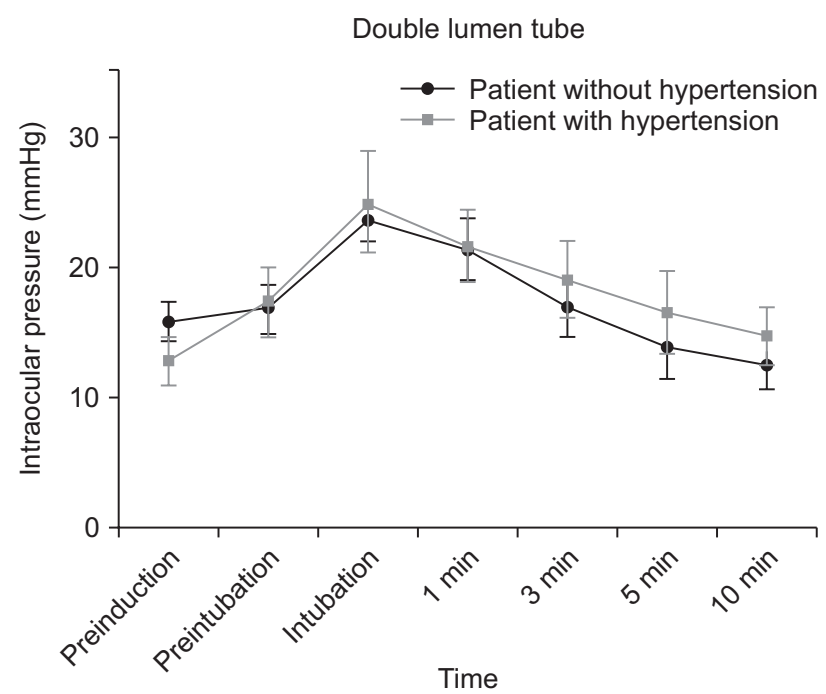

Fig. 4. Time course of intraocular pressure (IOP) for patients intubated with double lumen tube. There were no differences in IOPs between patients with and without hypertension. Symbols and error bars indicate means and SD, respectively. ery room.

There were no differences in the preoperative baseline IOP and OPP among groups. In patients without HTN, patients who were intubated with a DLT showed a higher IOP immediately and 1, 3, and 5 min after tracheal intubation, compared to those intubated with an SLT (Fig. 1). Importantly, IOP at T3 was $7.9 \mathrm{mmHg}$ higher than that at T1 in the DLT/ without HTN group, while IOP at T3 was only $5.0 \mathrm{mmHg}$ higher than that at T1 in the SLT/without HTN group $(\mathrm{P}=$ 0.019 compared to the SLT/without HTN group). The results were similar in patients with HTN (Fig. 2). In the DLT/HTN group, IOP was increased by $12.2 \mathrm{mmHg}$ after intubation, compared to T1. This increase in IOP was less in the SLT/ HTN group, where IOP increase was only $4.9 \mathrm{mmHg}(\mathrm{P}=0.009$ compared to the SLT/HTN group).

HTN patients showed a slightly, statistically nonsignificantly higher IOP after tracheal intubation than normotensive patients in both the SLT and DLT groups (Figs. 3 and 4).

The changes in the OPP were similar for all groups. The OPP decreased at T2 compared to baseline values and increased at T3, T4, and T5. The magnitude of the increase after

Table 2. Time Course of Ocular Perfusion Pressure for Patients without Hypertension

\begin{tabular}{lccc}
\hline \multicolumn{1}{c}{ Variable } & Single lumen tube & Double lumen tube & P value \\
\hline Preinduction & $78.8 \pm 8.2$ & $78.9 \pm 8.6$ & 0.958 \\
Preintubation & $71.5 \pm 7.6$ & $72.9 \pm 9.2$ & 0.885 \\
Intubation & $92.5 \pm 8.8$ & $101 \pm 16.2$ & 0.201 \\
$1 \mathrm{~min}$ & $81.2 \pm 9.2$ & $91.7 \pm 17.2$ & 0.195 \\
$3 \mathrm{~min}$ & $72.1 \pm 8.8$ & $82.6 \pm 15.3$ & 0.098 \\
$5 \mathrm{~min}$ & $66.5 \pm 8.3$ & $76.4 \pm 12.4$ & 0.831 \\
$10 \mathrm{~min}$ & $68.8 \pm 8.8$ & $68.4 \pm 10.2$ & 0.937 \\
\hline
\end{tabular}

Values are presented as mean $\pm \mathrm{SD}$. Values are $\mathrm{mmHg}$ in scale. No significant difference was found between groups.

Table 3. Time Course of Ocular Perfusion Pressure for Patients with Hypertension

\begin{tabular}{lccc}
\hline \multicolumn{1}{c}{ Variable } & Single lumen tube & Double lumen tube & P value \\
\hline Preinduction & $79.5 \pm 10.2$ & $86.5 \pm 10.5$ & 0.665 \\
Preintubation & $72.4 \pm 9.6$ & $74 \pm 8.1$ & 0.921 \\
Intubation & $95.7 \pm 18.5$ & $108 \pm 20.1$ & 0.101 \\
1 min & $88.1 \pm 15.3$ & $106.4 \pm 18.8$ & 0.083 \\
3 min & $84 \pm 15.1$ & $101.1 \pm 20.2$ & 0.092 \\
5 min & $79.9 \pm 16.2$ & $94.5 \pm 15.5$ & 0.097 \\
10 min & $64 \pm 15.4$ & $81.1 \pm 14.8$ & 0.068 \\
\hline
\end{tabular}

Values are presented as mean \pm SD. Values are $\mathrm{mmHg}$ in scale. No significant difference was found between groups. 
Table 4. Time Course of Mean Blood Pressure for Patients without Hypertension

\begin{tabular}{lccc}
\hline \multicolumn{1}{c}{ Variable } & Single lumen tube & Double lumen tube & P value \\
\hline Preinduction & $93.6 \pm 9.1$ & $95.1 \pm 8.6$ & 0.835 \\
Preintubation & $87.7 \pm 11.6$ & $90 \pm 13.9$ & 0.667 \\
Intubation & $113 \pm 14.2$ & $124.2 \pm 18.3$ & 0.109 \\
1 min & $97.3 \pm 10.1$ & $103.8 \pm 17.7$ & 0.272 \\
$3 \mathrm{~min}$ & $84.5 \pm 9.9$ & $89.9 \pm 15.5$ & 0.315 \\
$5 \mathrm{~min}$ & $76.5 \pm 8.9$ & $90.8 \pm 11.6$ & 0.088 \\
$10 \mathrm{~min}$ & $78.9 \pm 8.1$ & $91.9 \pm 9.9$ & 0.411 \\
\hline
\end{tabular}

Values are presented as mean \pm SD. Values are $\mathrm{mmHg}$ in scale. No significant difference was found between groups.

tracheal intubation was comparable among groups (Tables 2 and 3). Blood pressures before and after tracheal intubation are listed in Tables 4 and 5.

\section{DISCUSSION}

IOP increased sharply after tracheal intubation, and the increase was greater in the DLT groups than in the SLT groups. Considering that we continuously administered sevoflurane and remifentanil, which have been shown to have alleviating effects on IOP in previous studies [6,7], the increase in IOP after tracheal intubation was clinically important.

There are various perioperative factors that are considered to influence IOP during tracheal intubation. Among these factors, hemodynamic maintenance, ventilation strategy, and anesthetic agents are manageable by the anesthesiologist. In this study, we tried to maintain intraoperative BIS to about 50 and MAP between $\pm 20 \%$ of baseline values by modulating the depth of anesthesia and the $\mathrm{EtCO}_{2}$ to $30-35 \mathrm{mmHg}$ by regulating the respiratory rate. By strictly adjusting factors that were manageable by the anesthesiologist, we were able to evaluate the effects of different types of endotracheal tubes and preoperative HTN on IOP.

Sympathetic stimuli and adrenergic responses during tracheal intubation cause an increase in IOP [1]. The responses are thought to be the result of oropharyngeal and laryngotracheal stimulations by manipulation of the direct laryngoscope and placement of tube in the trachea. The mechanism of IOP increase following tracheal intubation is related to venoconstriction, which leads to increased central venous pressure and resistance to the outflow of aqueous humor in the trabecular meshwork between the anterior chamber and
Table 5. Time Course of Mean Arterial Pressure for Patients with Hypertension

\begin{tabular}{lccc}
\hline \multicolumn{1}{c}{ Variable } & Single lumen tube & Double lumen tube & P value \\
\hline Preinduction & $94.9 \pm 9.2$ & $99.6 \pm 10.9$ & 0.378 \\
Preintubation & $89.7 \pm 11.4$ & $91.5 \pm 11$ & 0.921 \\
Intubation & $116.2 \pm 20.6$ & $133.2 \pm 24.6$ & 0.135 \\
$1 \mathrm{~min}$ & $105.7 \pm 15.3$ & $127.9 \pm 20.6$ & 0.053 \\
$3 \mathrm{~min}$ & $97.8 \pm 16.8$ & $120.5 \pm 20.1$ & 0.041 \\
$5 \mathrm{~min}$ & $90.6 \pm 14.2$ & $110.8 \pm 16.7$ & 0.079 \\
$10 \mathrm{~min}$ & $74.3 \pm 12.1$ & $96.1 \pm 11.9$ & 0.031 \\
\hline
\end{tabular}

Values are presented as mean $\pm \mathrm{SD}$. Values are $\mathrm{mmHg}$ in scale.

Schlemm's canal [8]. The reason that a DLT was associated with a large increase in IOP during tracheal intubation in our study can be explained as follows: the external diameter of a DLT is larger than that of an SLT, therefore, intubation with a DLT is considered more difficult and more stimulating to the trachea, which leads to higher sympathetic responses than intubation with an SLT.

Previous studies have not reported a correlation between systemic HTN and the increase in IOP [4,9-11]. Our hypothesis was that IOP would be relevant to the presence of HTN because of the influence of vascular dysregulation or abnormal blood pressure on ocular perfusion [12]. However, there was no relationship between IOP and systemic HTN, which is consistent with Czarnik et al. [13]. Alternatively, Mitchell et al. [10] reported that poorly controlled HTN increased the risk for open-angle glaucoma, whereas controlled HTN did not. Therefore, our findings indicate that all patients with underlying HTN were well-managed with antihypertensive agents, and anesthetics for induction and maintenance led to a lower IOP [14], offsetting the increase in IOP caused by HTN.

Most postoperative visual impairments do not involve direct pressure on the periorbital area or the globe itself, but rather changes in blood flow to the eyeball or optic nerve, caused by either a decrease in perfusion or an embolism [15]. In predicting the perfusion to the eyeball or optic nerve, OPP is commonly used as a variable. OPP, defined as the difference between the MAP and IOP, can be decreased by an increase in IOP, despite the maintenance of a normal MAP. It is therefore important to understand how IOP and OPP change in anesthetized patients during and after tracheal intubation. In our study, there were no significant differences in OPP between the SLT and DLT groups. This result is inconsistent with our hypothesis that DLT would significantly 
increase IOP and thus negatively influence OPP. The reason for maintained or even higher OPP for both the DLT and SLT groups in spite of an IOP increase during tracheal intubation is because the MAP was elevated during tracheal intubation, which offset the negative effects of an increase in IOP on the OPP. To maintain OPP and prevent postoperative ophthalmic complications, it is important for an anesthesiologist to maintain the proper MAP by regulating fluid administration and depth of anesthesia, as well as to prevent the increase in IOP during anesthesia.

We did not observe an increased incidence of ophthalmic complications by tracheal intubation using a DLT. No patients complained of postoperative ophthalmic complications despite IOP increasing significantly during and after tracheal intubation using a DLT. This was probably because the duration of IOP increase during tracheal intubation in our study was only approximately $3 \mathrm{~min}$, so the OPP was properly maintained because of the increase in the MAP. Ophthalmic complications such as visual loss may occur as a result of sustained IOP increases during surgery [16,17]. Maintaining IOP over the normal range is very important because of the potential for optic nerve damage, and the increasing frequency of normal-tension glaucoma patients. It is therefore very important to actively control IOP [18,19]. An increase in IOP should especially be avoided in patients with penetrating eye surgery in glaucomatous or elderly patients, in whom the control of IOP is of paramount concern.

This study had some limitations. The patients in the HTN groups (SLT/HTN and DLT/HTN groups) were patients with well-controlled HTN; hence, the results from these data cannot be extrapolated to other groups of patients. Because poorly controlled HTN is associated with a risk for open-angle glaucoma [10], the possibility of severe and sustained increases in IOP during and after tracheal intubation in patients with poorly controlled HTN should be avoided. Second, this study was conducted in the context of a rapid-sequence induction using succinylcholine. The results cannot be generalized for regular intubation situations using non-depolarizing neuromuscular blockers. Third, because this study was not a randomized trial, it is important to recognize that IOP after tracheal intubation may be further affected by surgery or the characteristics of patients. A large-scale randomized trial involving more patients and surgery is therefore required.

In conclusion, tracheal intubation using a DLT increased
IOP more than intubation using an SLT in rapid sequence intubation with succinylcholine, and well-controlled underlying HTN did not increase IOP during tracheal intubation.

\section{CONFLICTS OF INTEREST}

No potential conflict of interest relevant to this article was reported.

\section{ORCID}

Hojun Ro: https://orcid.org/0000-0002-5959-0098

\section{REFERENCES}

1. Lamb K, James MF, Janicki PK. The laryngeal mask airway for intraocular surgery: effects on intraocular pressure and stress responses. Br J Anaesth 1992; 69: 143-7.

2. Hamp T, Stumpner T, Grubhofer G, Ruetzler K, Thell R, Hager H. Haemodynamic response at double lumen bronchial tube placement - Airtraq vs. MacIntosh laryngoscope, a randomised controlled trial. Heart Lung Vessel 2015; 7: 54-63.

3. Mizrak A, Kocamer B, Deniz H, Yendi F, Oner U. Cardiovasular changes after placement of a classic endotracheal tube, doublelumen tube, and Laryngeal Mask Airway. J Clin Anesth 2011; 23: 616-20.

4. Vaajanen A, Mervaala E, Oksala O, Vapaatalo H. Is there a relationship between blood pressure and intraocular pressure? An experimental study in hypertensive rats. Curr Eye Res 2008; 33: 325-32.

5. Beneyto P, Barajas MA, Garcia-de-Blas F, Del Cura I, Sanz T, Vello $\mathrm{R}$, et al. Predictive value of tonometry with Tono-pen XL in primary care. Br J Gen Pract 2007; 57: 653-4.

6. Tuzcu K, Tuzcu EA, Karcioglu M, Davarci I, Coskun M, Ilhan O, et al. The effects of remifentanil and esmolol on increase in intraocular pressure due to laryngoscopy and tracheal intubation: a double-blind, randomized clinical trial. J Glaucoma 2015; 24: 372-6.

7. Sator-Katzenschlager S, Deusch E, Dolezal S, Michalek-Sauberer A, Grubmüller R, Heinze G, et al. Sevoflurane and propofol decrease intraocular pressure equally during non-ophthalmic surgery and recovery. Br J Anaesth 2002; 89: 764-6.

8. Murphy DF. Anesthesia and intraocular pressure. Anesth Analg 1985; 64: 520-30.

9. Klein BE, Klein R, Knudtson MD. Intraocular pressure and systemic blood pressure: longitudinal perspective: the Beaver Dam 
Eye Study. Br J Ophthalmol 2005; 89: 284-7.

10. Mitchell P, Lee AJ, Rochtchina E, Wang JJ. Open-angle glaucoma and systemic hypertension: the blue mountains eye study. J Glaucoma 2004; 13: 319-26.

11. Xu L, Wang H, Wang Y, Jonas JB. Intraocular pressure correlated with arterial blood pressure: the beijing eye study. Am J Ophthalmol 2007; 144: 461-2.

12. Gherghel D, Hosking SL, Orgül S. Autonomic nervous system, circadian rhythms, and primary open-angle glaucoma. Surv Ophthalmol 2004; 49: 491-508.

13. Czarnik T, Gawda R, Kolodziej W, Latka D, Sznajd-Weron K, Weron R. Associations between intracranial pressure, intraocular pressure and mean arterial pressure in patients with traumatic and non-traumatic brain injuries. Injury 2009; 40: 33-9.

14. Ding C, Wang P, Tian N. Effect of general anesthetics on IOP in elevated IOP mouse model. Exp Eye Res 2011; 92: 512-20.
15. Stambough JL, Dolan D, Werner R, Godfrey E. Ophthalmologic complications associated with prone positioning in spine surgery. J Am Acad Orthop Surg 2007; 15: 156-65.

16. Pinkney TD, King AJ, Walter C, Wilson TR, Maxwell-Armstrong C, Acheson AG. Raised intraocular pressure (IOP) and perioperative visual loss in laparoscopic colorectal surgery: a catastrophe waiting to happen? A systematic review of evidence from other surgical specialities. Tech Coloproctol 2012; 16: 331-5.

17. Nickels TJ, Manlapaz MR, Farag E. Perioperative visual loss after spine surgery. World J Orthop 2014; 5: 100-6.

18. Trivli A, Koliarakis I, Terzidou C, Goulielmos GN, Siganos CS, Spandidos DA, et al. Normal-tension glaucoma: pathogenesis and genetics. Exp Ther Med 2019; 17: 563-74.

19. Razeghinejad MR, Lee D. Managing normal tension glaucoma by lowering the intraocular pressure. Surv Ophthalmol 2019; 64: 111-6. 